\title{
APRENDIZAJE ARTIFICIAL DE SEÑALES EEG DE APOYO EN LA GENERACIÓN DE ÓRDENES CORPORALES EN PERSONAS CON FALENCIA MOTORA.
}

\author{
${ }^{1}$ Raul Eduardo Huarote Zegarra dol $^{\text {dol }}$ Alfredo Cesar Larios Franco, ${ }^{1}$ Angel \\ Fernando Navarro Raymundo, ${ }^{1}$ Julio Elvis Valero Cajahuanca, ${ }^{1}$ Janett Deisy Julca \\ Flores \\ ${ }^{1}$ Universidad Nacional Tecnológica de Lima Sur, Lima, Perú
}

Recibido: 05/09/2021 Revisado: 03/10/2021 Aceptado: 16/11/2021 Publicado: 30/01/2022

\begin{abstract}
Resumen
La presente investigación se trata de que, a partir de las diferentes pruebas realizadas en base a datos obtenidos de tipo señales de frecuencias electroencefalograma (EEG), desde un dispositivo OpenBCI, queremos detectar la actividad eléctrica característica o particular del cerebro (específicamente para generar ordenes), mediante pequeños discos metálicos o electrodos, este dispositivo se fija en el cuero cabelludo y se obtiene mediante un sensor de frecuencias. Saber que las neuronas del cerebro se comunican a través de impulsos eléctricos y están activas en todo momento, incluso mientras duermes. Esta actividad eléctrica se manifiesta como líneas onduladas en un registro de EEG, este insumo permite realizar pruebas a partir de los patrones característicos, así como del proceso de aprendizaje de dichos patrones en base a una red neuronal artificial de tipo backpropagation, mostrando los resultados de entrenamiento y las pruebas en un entorno colab y con un lenguaje de programación Python 3.9.
\end{abstract}

Palabras claves: red neuronal, órdenes, openbci, electroencefalograma.

Abstract. This research is about the fact that, from the different tests carried out based on data obtained such as electroencephalogram (EEG) frequency signals, from an OpenBCI device, we want to detect the characteristic or particular electrical activity of the brain (specifically to generate orders), by means of small metal discs or electrodes, this device is fixed on the scalp and is obtained by means of a frequency sensor. Know that neurons in the brain communicate through electrical impulses and are active at all times, even while you sleep. This electrical activity is manifested as wavy lines in an EEG record, this input allows tests to be carried out from the characteristic patterns, as well as the learning process of said patterns based on an 
artificial neural network of the backpropagation type, showing the results of training and testing in a collaborative environment and with a Python 3.9 programming language.. Keywords: neural network, order, openBCI, electroencephalogram.

\section{Introducción}

En la presente investigación lo que se trata es que a partir de las diferentes pruebas realizadas con un dispositivo electroencefalograma (EEG), se quiere detectar la actividad eléctrica característica o particular del cerebro mediante pequeños discos metálicos o electrodos para generar ordenes, tales como derecho, izquierda, arriba y abajo, el cual este dispositivo está fijado sobre el cuero cabelludo. Sabiendo que las neuronas cerebrales se comunican a través de impulsos eléctricos y están activas en todo momento, incluso mientras duermes. Esta actividad eléctrica se manifiesta como líneas onduladas en un registro de electroencefalograma. Encontramos notables investigaciones, como [1] tiene una fuente de información, acerca de los parámetros de EEG en estado de reposo considerando para ello los registros después del accidente cerebrovascular encontrando unos deterioros y predecir dichos deterioros en el motor de las extremidades superiores reflejado por la puntuación motora de Fugl-Meyer (FM-UE), después de seis meses, y si tienen valor pronóstico además de FM-UE al inicio del estudio. También [2] concluye que en promedio la precisión en la detección de emociones en la base de datos de SEED para el enfoque contingente del sujeto se mejora en un 4,12\%, para el enfoque no contingente en un 4,5\% y para el enfoque interdependiente mejora en un 1,3\%. Para reconfirmar los hallazgos anteriores, se realiza un experimento más para el enfoque no contingente de sujetos en la base de datos DEAP. En la base de datos DEAP para el experimento sujeto no contingente, la precisión promedio se mejora en un 7.04\%. Los resultados experimentales del extractor de características propuesto LF-DfE con la red BiLSTM han mejorado con respecto a los métodos existentes. Así también [3] en su investigación sobre la clasificación demuestran que la mentira se puede diferenciar de decir la verdad con una precisión del 86,25\% con el método de dejar fuera a una persona, a partir de la evaluación del EEG. [4] ha encontrado evidencia adicional en apoyo de un papel funcional de la actividad alfa durante el desarrollo temporal del proceso DT, destacando el papel clave del aumento de poder 
alfa sobre la corteza frontal, central, temporal y parietal para la producción de respuestas alternativas originales en AUT. Cabe resaltar que [5] menciona que el pensamiento creativo es fundamental para las artes, las ciencias y la vida cotidiana, por tanto es necesario se puede analizar estas acciones y basarse una investigación a partir de estos datos, también [6] logra identificar la asociación el procesamiento interno activo y la creatividad. Así también [7] tuvo como objetivo investigar las bases neuronales de los procesos involucrados en la superación de los efectos de fijación durante la generación de ideas creativas, utilizando la tarea AU adaptada para la grabación de EEG, como [8] logró implementar en MATLAB multiplataforma (The Mathworks, Inc.) para procesar colecciones de datos de EEG de prueba única y / o promediados de cualquier número de canales. También [9] sostiene que en revisiones recientes, parece haber pruebas sólidas de que el poder alfa del EEG es particularmente sensible a las diversas demandas relacionadas con la creatividad e involucradas en la ideación creativa. Así [10] menciona que la actividad cortical en la banda alfa del EEG ha demostrado ser particularmente sensible a las demandas relacionadas con la creatividad, pero su significado funcional en el contexto de la cognición creativa aún no se ha aclarado. Como [11] estudió la actividad de EEG en bailarines profesionales $(n=15)$ que han alcanzado un alto nivel de experiencia en este dominio. Este grupo se comparó con un grupo de novatos $(\mathrm{n}=17)$ que solo tienen una experiencia básica en el baile y no completaron una formación integral en este campo. Una vez más confirma [12] que en la actividad alfa indican estados cerebrales están orientados interna y externamente, también en diferentes parámetros de actividad alfa oscilatoria predicen la percepción visual y se presenta un modelo neurocognitivo para explicar el parpadeo atencional. También [13] tiene en cuenta que los componentes de la representación es una combinación lineal de las variables originales y que los métodos de transformación lineal bien conocidos incluyen el análisis de componentes principales, el análisis factorial y la búsqueda de proyecciones. [14] Examinó los efectos neurofisiológicos y que también se pueden encontrar cuando ambos modos de procesamiento cognitivo (convergente frente a divergente) se evalúan mediante la misma tarea empleando una variación simple de instrucción, para ello tuvo una muestra de 55 participantes que realizó la tarea de usos alternativos, así como una tarea de asociación de palabras más básica mientras se registraba el EEG para evidenciar diferentes patrones de actividad alfa de EEG relacionados con modos convergentes y divergentes de 
procesamiento de tareas. A partir de estas investigaciones refuerza y motiva para realizar la detección de patrones característicos para generar órdenes.

\section{Materiales y métodos.}

La presente investigación se ha considerado como parte de materiales el lector OpenBCI, y a partir de este dispositivo se obtiene un insumo llamado electro encefalograma y que este conjunto de datos, que no es más que un conjunto de valuaciones que representa unas ondas. Posteriormente se va a procesar, aplicando la inteligencia artificial, específicamente las redes neuronales artificiales.

\subsection{Método para identificar frecuencias características de EEG y representarlo en patrones}

Cabe resaltar que las frecuencias realizadas, es para poder tener un análisis especifico de que datos se puede realizar como patrones de entrada, teniendo en cuenta que los resultados se van a representar en función de las ondas, por tanto según la base las ondas Delta están en un rango de 0.2-4 Hz por tanto se consideran las más lentas y es predominante cuando está en sueño, las ondas theta están en un rango de 4-8 Hz, las ondas alpha están en un rango de 8-12 Hz, las ondas Beta están en un rango de 12-30 Hz y las ondas Gamma están en un rango de 30-90 Hz, tal como se visualiza en la Fig. 1.

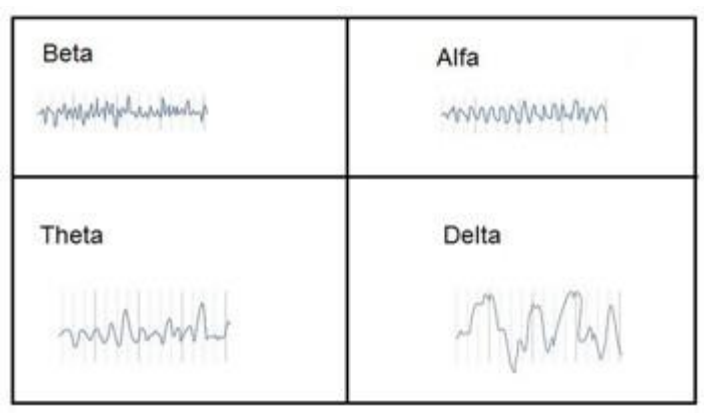

Fig. 1: Representación de las ondas Gamma, Beta, Alpha, theta y delta.

Tener en cuenta que necesariamente se ha tenido que realizar las operaciones bajo las condiciones de que esta con los ojos cerrados, es una persona joven (21 años), no tiene BIOTECH \& ENGINEERING Untels. Ene -Jun.2(1), 2022; ISSN:2788 -4295; 282-292 
condiciones especifica de impedimento, y la muestra de adquisición se ha dado muestra en la Fig. 2.

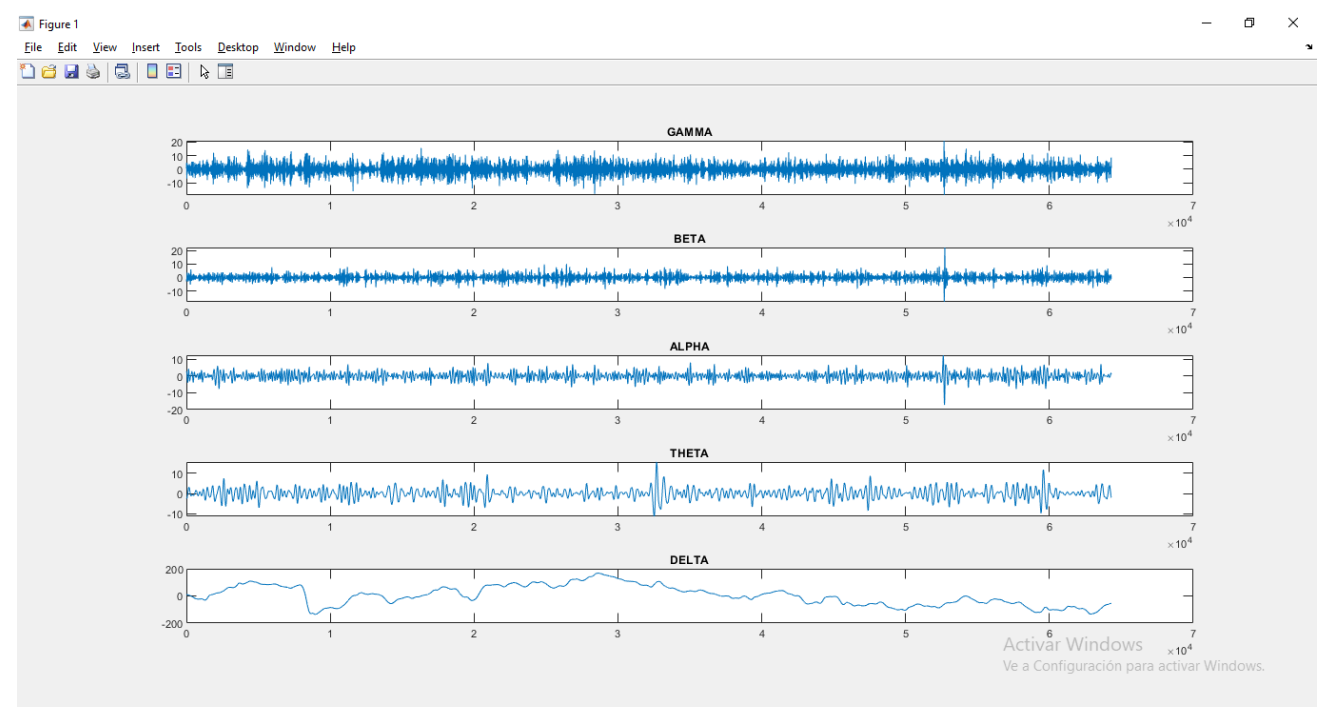

Fig 2: Ondas Gamma, Beta, Alpha, theta y delta representados gráficamente y obtenidas por el OpenBCI, del movimiento de la mano izquierda.

\subsection{Modelado de la red neuronal artificial para el aprendizaje de las frecuencias}

Los datos como frecuencias EEG, se va a exportar a formato.csv para poder crear y realizar las pruebas necesarias para definir la estructura de la red neuronal artificial en un entorno libre de tipo COLAB.

Una vez extraídas características de las señales en estudio en formato manejable para un entorno de la red neuronal, estas son una representación más compacta de la información que contienen, sin embargo, se requiere de una herramienta que reconozca estas características que diferencian una señal patológica de una no patológica, para esta clasificación las redes neuronales son un medio eficiente que permite con muy buena exactitud reconocer cuando un conjunto de características, provienen de una señal patológica o una señal normal. 
Es por esto y otros motivos que serían interesante simular una red neuronal con unas wavelets como las biortogonales, puesto que se ha probado en otros trabajos su eficiencia en el análisis y síntesis de señales EEG. También convendría realizar un análisis de los coeficientes de más de un nivel de aproximación puesto que algunas de las diferencias más significativas encontradas en este trabajo tuvieron lugar entre los coeficientes del nivel de aproximación A5. Por otro lado, y en vista del poder de clasificación que poseen las redes neuronales sería útil estudiar otras estructuras multicapa en una red neuronal y comparar los resultados entre estas estructuras y así obtener una clasificación cada vez más eficiente.

En la Fig 3, muestra las características comunes para ser analizados en la línea de base (Baseline), y en función de estos datos, hay que tomar en como característica de la los datos para realizar la estructura de la red neuronal.

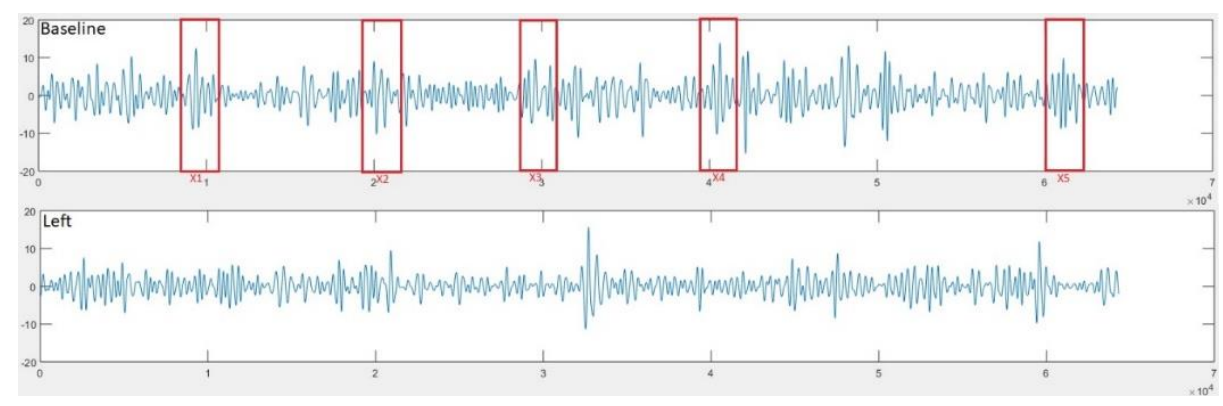

Fig 3: Características comunes encontradas para el aprendizaje.

Las características de la red neuronal está sujeta a la cantidad de datos encontrados, por tanto la estructura de la red neuronal está establecida de la siguiente manera, con los 272 patrones de entrada, 2 capas ocultas y una capa de salida con 3 neuronas, tal como se visualiza en la Fig 4. 


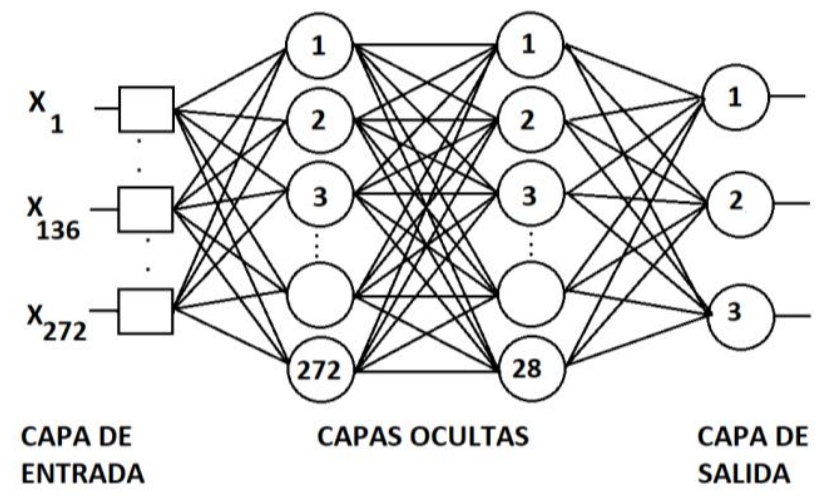

Fig. 4: Estructura de la red neuronal artificial back propagation para el aprendizaje.

\subsection{Aplicación del modelo}

El código donde se realizar el proceso de aprendizaje como prueba de la red neuronal establecida está en la Fig 5, donde se realiza la adquisición de datos, la estructura de la red neuronal artificial, según el modelo aplicado en la Fig 4, el entrenamiento con 100 épocas, para posterior evaluación de los resultados en el mapeo. 


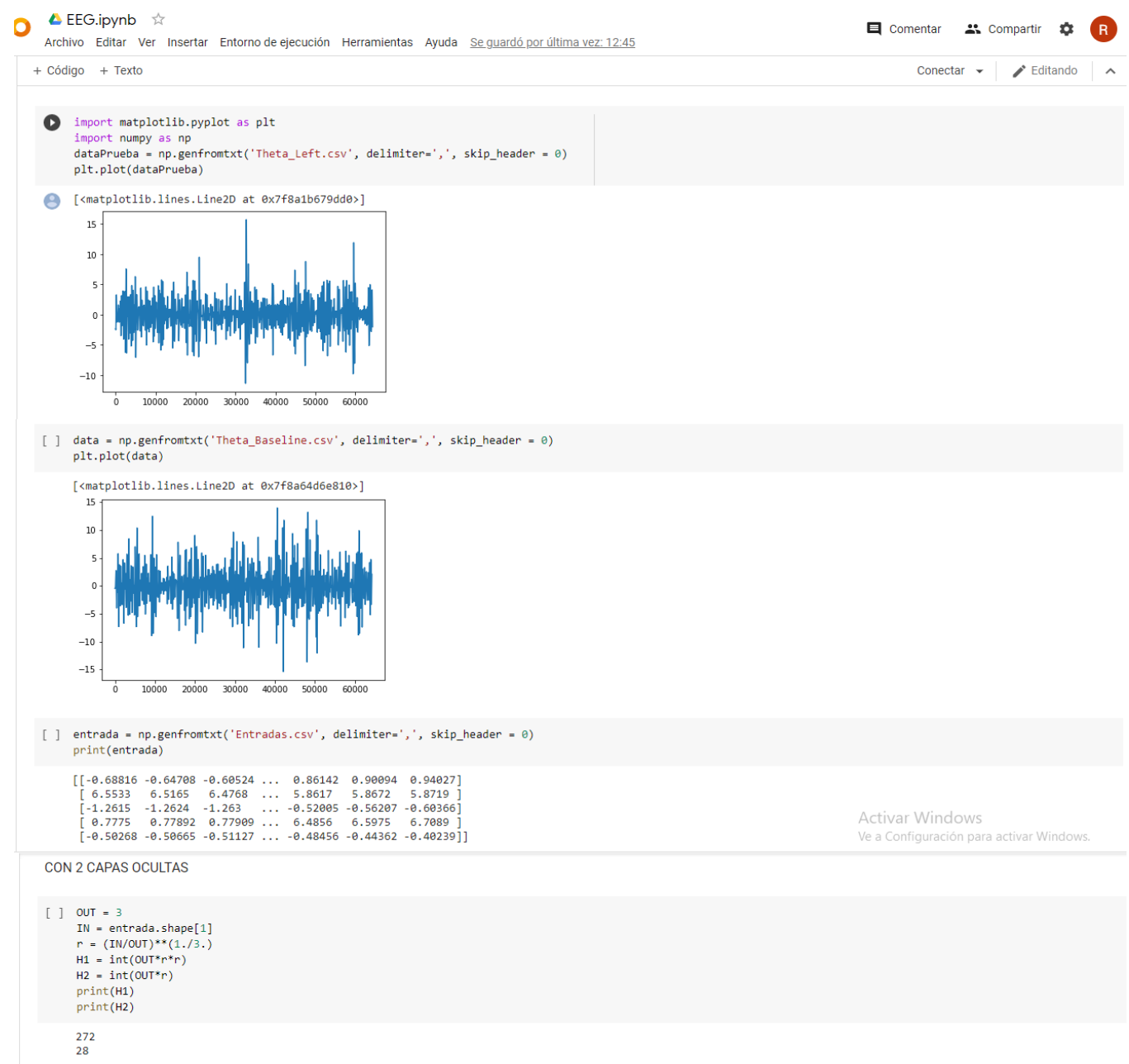

\section{ENTRENAMIENTO DE LA RED NEURONAL}

(1) from keras.models import Sequential

import Dense

$Y=[[\theta, \theta, \theta],[\theta, \theta, 1],[\theta, 1, \theta],[\theta, 1,1],[1, \theta, \theta]]$

datoEntredas $=n p \cdot$ array (entrada, "float32")

model $=$ Sequential()

model. add(Dense(units=H1, input_dim=IN, activation='relu'))

\#model. add (Dense(H1, activation='relu'))

mode1.add(Dense(

model.compile(loss='binary_crossentropy', optimizer='Adam', ,metrics=[' binary_accuracy'])

datos $=$ model.fit(datoEntredas, datosalida, epochs $=100$ )

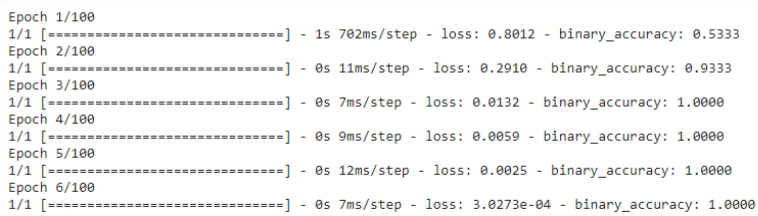

Fig 5: Código fuente de la estructura de la red neuronal y el entrenamiento. 


\section{RESULTADOS}

Los resultados al aplicar la red neuronal en un entorno colab, se muestran en la siguiente Fig 6, donde se tiene que etiquetar cada uno de los resultados encontrados o patrones característicos dentro del EEG.

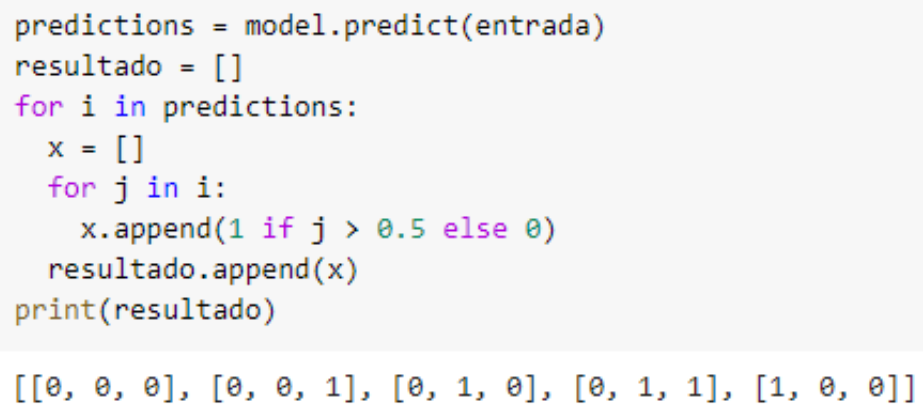

Fig 6: Resultados de las pruebas al implementar la red neuronal artificial para las pruebas de los datos del EEG.

\section{Discusión.}

Los resultados obtenidos correspondientes a los datos del electroencefalograma, y el mapeo realizado con las pruebas de los mismos datos, tal como lo refleja en la Fig. 6, se verifica que se obtiene datos que pueden representar una orden o una intensión de orden, y en función del mismo modelo de la red. Cabe resaltar que para el mapeo se ha realizado con los mismos datos de entrenamiento.

\section{Conclusión.}

Por tanto podemos concluir que usando la red neuronal artificial de tipo backpropagation, si logra aprender patrones característicos de un conjunto de frecuencias que representa una orden en particular. A su vez también se puede tomar como base para futuras investigaciones, ya que se puede tomar estos insumos que es las frecuencias para usarlas en otro tipo de red neuronal, así para clasificarla, como usar un 
modelo estadístico de análisis factorial de correspondencia múltiple, para ver los factores que influyen en un conjunto de sucesos representados en el electroencefalograma.

\section{Referencias bibliográficas}

[1] Mique Saes, Carel G.M. Meskers, Andreas Daffertshofer, Erwin E.H. van Wegen, Gert Kwakkel, on behalf of the 4D-EEG consortium. 2020. Are early measured resting-state EEG parameters predictive for upper limb motor impairment six months poststroke?. Clinical Neurophysiology Volume 132, Issue 1, January 2021, Pages 56-62. DOI: https://doi.org/10.1016/j.clinph.2020.09.031

[2] Vaishali M.Joshi and Rajesh B.Ghongade. 2021. EEG based emotion detection using fourth order spectral moment and deep learning. Biomedical Signal Processing and Control. Volume 68, July 2021, 102755. DOI: https://doi.org/10.1016/j.bspc.2021.102755

[3] Marzieh Daneshi Kohan, AliMotie Nasrabadi, Alisharifi, Mohammad Bagher Shamsollahi. 2019. Interview based connectivity analysis of EEG in order to detect deception. Medical $\begin{array}{lllll}\text { Hypotheses. } & \text { Volume } & \text { 136, } & \text { March } & \text { DOI: }\end{array}$ https://doi.org/10.1016/j.mehy.2019.109517

[4] Sergio Agnoli, Marco Zanon, Serena Mastria, Alessio Avenanti, Giovanni Emanuele Corazza. 2019. Predicting response originality through brain activity: An analysis of changes in EEG alpha power during the generation of alternative ideas. NeuroImage. Volume 207, 15 February 2020, 116385. DOI: https://doi.org/10.1016/j.neuroimage.2019.116385

[5] Roger E. Beaty, Mathias Benedek, Paul J. Silvia, Daniel L. Schacter. 2015. Creative Cognition and Brain Network Dynamics. Trends in Cognitive Sciences. Volume 20, Issue 2, February 2016, Pages 87-95. DOI: https://doi.org/10.1016/j.tics.2015.10.004

[6] Benedek et al., 2011. M. Benedek, S. Bergner, T. Koenen, A. Fink, A.C. Neubauer. EEG alpha synchronization is related to top-down processing in convergent and divergent thinking Neuropsychologia, 49 (2011), pp. 3505-3511, 10.1016/j.neuropsychologia.2011.09.004 
[7] A. Camarda, É. Salvia, J. Vidal, B. Weil, N. Poirel, O. Houdé, et al. Neural basis of functional fixedness during creative idea generation: an EEG study Neuropsychologia, 118 (2018), pp. 4-12, 10.1016/j.neuropsychologia.2018.03.009.

[8] A. Delorme, S. Makeig. EEGLAB: an open source toolbox for analysis of single-trial EEG dynamics including independent component analysis. J. Neurosci. Methods, 134 (1) (2004), pp. 9-21, 10.1016/j.jneumeth.2003.10.009

[9] A. Fink, M. Benedek. EEG alpha power and creative ideation

Neurosci. Biobehav. Rev., 44 (2014), pp. 111-123, 10.1016/j.neubiorev.2012.12.002

[10] A. Fink, R.H. Grabner, M. Benedek, G. Reishofer, V. Hauswirth, M. Fally, C. Neuper, F. Ebner, A.C. Neubauer. The creative brain: investigation of brain activity during creative problem solving by means of EEG and fMRI. Hum. Brain Mapp., 30 (2009), pp. 734-748, 10.1002/hbm.20538.

[11] A. Fink, B. Graif, A.C. Neubauer. Brain correlates underlying creative thinking: EEG alpha activity in professional vs. novice dancers. Neuroimage, 46 (2009), pp. 854-862, 10.1016/j.neuroimage.2009.02.036.

[12] S. Hanslmayr, J. Gross, W. Klimesch, K.L. Shapiro. The role of alpha oscillations in temporal attention. Brain Res. Rev., 67 (2011), pp. 331-343, 10.1016/j.brainresrev.2011.04.002.

[13] A. Hyvärinen, E. Oja. Independent component analysis: algorithms and applications. Neural Netw., 13 (4-5) (2000), pp. 411-430, 10.1016/S0893-6080(00)00026-5

[14] E. Jauk, M. Benedek, A.C. Neubauer. Tackling creativity at its roots: evidence for different patterns of EEG alpha activity related to convergent and divergent modes of task processing. Int. J. Psychophysiol., 84 (2012), pp. 219-225, 10.1016/j.ijpsycho.2012.02.012 